\title{
Polarizing the Nazarov cyclization: Efficient Catalysis under Mild Conditions
}

\author{
Wei He, Xiufeng Sun, and Alison J. Frontier* \\ Department of Chemistry, University of Rochester, Rochester, NY, 14627
}

\section{Supporting information}

General Methods: All reactions were carried out in vials without argon protection ${ }^{1}$. $\mathrm{Cu}(\mathrm{OTf})_{2}$ was stored in glove box before use. Dichloroethane and dichloromethane were purchased from Fisher and distilled over calcium hydride. Column chromatography was performed on EM Science silica gel 60 (230-400 mesh). Visualization was done with potassium permanganate or ceric ammonium molybdate.

Infared spectra were recorded on a ATI Mattson Genesis FT-IR spectrometer. ${ }^{1} \mathrm{H}$ NMR and ${ }^{13} \mathrm{C}$ NMR spectra were collected on a Bruker Amax 400 or an Avance $400 \mathrm{MHz}$ spectrometer at ambient temperature. Chemical shift $\delta$ was reported in unit of parts per million downfield from tetramethylsilane. Elemental Analysis was done by MHW labs at Phoenix, AZ. HRMS was done on a ThermoFinnigan MAT 95XL with electrospray with $50 \% \mathrm{MeOH}$.

\section{Preparation of substrates}

All Nazarov substrates bearing ester group were prepared from Knoevenagel reaction between a $\beta$-keto ester which already contains the left olefin moiety and an aldehyde ${ }^{2}$. Substrate 15a, 15f, 17a and 17f were prepared from condensation between a ketone which already contains the left olefin moiety and the corresponding aldehyde under basic conditions.

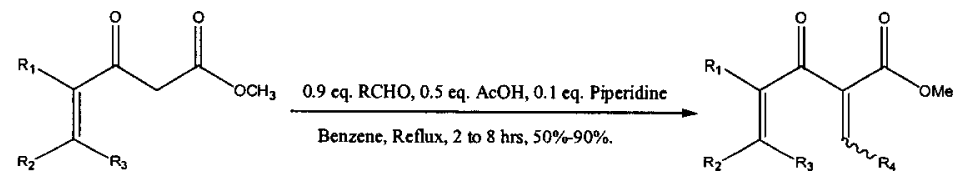

\section{Spectroscopic data of substrates:}

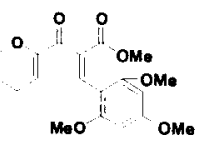

2-(5,6-Dihydro-4H-pyran-2-carbonyl)-3-(2,4,6-trimethoxy-phenyl)-acrylic acid methyl ester 3a $11 \mathrm{NMR}\left(400 \mathrm{MHz}, \mathrm{CDCl}_{3}\right) \delta 7.90(\mathrm{~s}, 1 \mathrm{H}), 5.98(\mathrm{~s}, 2 \mathrm{H}), 5.79(\mathrm{~s}, 1 \mathrm{H}), 3.98(\mathrm{t}, J=4.6 \mathrm{~Hz}, 2 \mathrm{H}), 3.74(\mathrm{~s}$,

\footnotetext{
1 While argon protection did not generally improve yield for most substrates, after submission we found that argon pretection of fairly unreactive substrates $15 f$ and $17 f$ gave better yields.

${ }^{2}$ Desimoni, G. , Faita, G. , Ricci, M, Righetti, P. , Tetrahedron 1998, 54, 9581
} 
$3 \mathrm{H}), 3.71(\mathrm{~s}, 3 \mathrm{H}), 3.66(\mathrm{~s}, 6 \mathrm{H}), 2.06(\mathrm{~d}, J=4.5 \mathrm{~Hz}, 2 \mathrm{H}), 1.75(\mathrm{t}, J=4.4 \mathrm{~Hz}, 2 \mathrm{H}) ;{ }^{13} \mathrm{C} \mathrm{NMR}\left(\mathrm{CDCl}_{3}, 100\right.$ MHz) $\delta 188.6,166.7,163.4,159.3,151.1,135.3,127.5,111.1,104.8,90.0,66.1,55.2,54.9,51.8,21.6$, 20.6; IR ( $\mathrm{NaCl}$ plate, $\left.\mathrm{cm}^{-1}\right): 1712,1603,1460,1336,1255,1206,1156,1127,1056$; Elemental analysis calculated for $\mathrm{C}_{19} \mathrm{H}_{22} \mathrm{O}_{7}$ : C 62.97\%; H 6.12\%. Found : C 62.79\%; H 6.20\%.

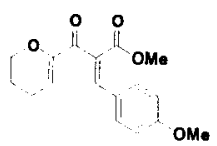

2-(5,6-Dihydro-4H-pyran-2-carbonyl)-3-(4-methoxy-phenyl)-acrylic acid methyl ester 3b ${ }^{\mathrm{H}} \mathrm{H}$ NMR $\left(400 \mathrm{MHz}, \mathrm{CDCl}_{3}\right) \delta 7.59(\mathrm{~s}, 1 \mathrm{H}), 7.16(\mathrm{~d}, J=8.8 \mathrm{~Hz}, 2 \mathrm{H}), 6.67(\mathrm{~d}, J=8.8 \mathrm{~Hz}, 2 \mathrm{H}), 5.87(\mathrm{t}$, $J=4.4 \mathrm{~Hz}, 1 \mathrm{H}), 3.93(\mathrm{t}, J=5.6 \mathrm{~Hz}, 2 \mathrm{H}), 3.62(\mathrm{~s}, 6 \mathrm{H}), 1.98(\mathrm{~m}, 2 \mathrm{H}), 1.65(\mathrm{t}, J=6 \mathrm{~Hz}, 2 \mathrm{H}) ;{ }^{13} \mathrm{C} \mathrm{NMR}$ $\left(\mathrm{CDCl}_{3}, 100 \mathrm{MHz}\right) \delta 190.7,165.3,161.2,150.9,142.5,132.0,127.1,125.2,116.2,114.0,66.3,55.1,52.0$, 21.0, 20.8; IR ( NaCl plate, $\mathrm{cm}^{-1}$ ): 1714, 1601, 1512, 1259, 1204, 1175, 734; HRMS calculated for $\mathrm{C}_{17} \mathrm{H}_{18} \mathrm{O}_{5}$ $(\mathrm{M}+\mathrm{H})^{+}$303.1224, Found : 303.1227 .

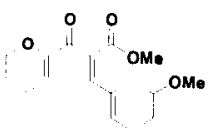

2-(5,6-Dihydro-4H-pyran-2-carbonyl)-3-(3-methoxy-phenyl)-acrylic acid methyl ester $3 c$ 'H NMR $\left(400 \mathrm{MHz}, \mathrm{CDCl}_{3}\right) \delta 7.58(\mathrm{~s}, 1 \mathrm{H}), 7.03(\mathrm{t}, \mathrm{J}=3.6 \mathrm{~Hz}, 1 \mathrm{H}), 6.75(\mathrm{~d}, J=9.6 \mathrm{~Hz}, 1 \mathrm{H}), 6.69$ (s, $2 \mathrm{H}), 5.81(\mathrm{t}, J=4.4 \mathrm{~Hz}, 1 \mathrm{H}), 3.86(\mathrm{t}, J=4.6 \mathrm{~Hz}, 2 \mathrm{H}), 3.58(\mathrm{~s}, 3 \mathrm{H}), 3.53(\mathrm{~s}, 3 \mathrm{H}), 1.94(\mathrm{~d}, J=6 \mathrm{~Hz}, 2 \mathrm{H})$, $1.57(\mathrm{t}, J=6.0 \mathrm{~Hz}, 2 \mathrm{H}) ;{ }^{13} \mathrm{C} \mathrm{NMR}\left(\mathrm{CDCl}_{3}, 100 \mathrm{MHz}\right) \delta 189.9,164.8,159.3,150.9,142.6,133.9,130.3$, $129.5,122.4,116.4,115.8,114.3,66.2,54.7,52.1,21.0,20.7$; IR (NaCl plate, $\left.\mathrm{cm}^{-1}\right): 1715,1671,1624$, 1236, 1051; HRMS calculated for $\mathrm{C}_{17} \mathrm{H}_{18} \mathrm{O}_{5}(\mathrm{M}+\mathrm{H})^{+} 303.1233$ Found : 303.1227 .

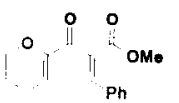

\section{2-(5,6-Dihydro-4H-pyran-2-carbonyl)-3-phenyl-acrylic acid methyl ester}

3d ${ }^{1} \mathrm{H}$ NMR $\left(400 \mathrm{MHz}, \mathrm{CDCl}_{3}\right) \delta 7.58(\mathrm{~s}, 1 \mathrm{H}), 7.13(\mathrm{~m}, 2 \mathrm{H}), 7.04(\mathrm{~m}, 3 \mathrm{H}), 5.77(\mathrm{t}, J=4.1 \mathrm{~Hz}, 1 \mathrm{H}), 3.79$ $(\mathrm{t}, J=4.4 \mathrm{~Hz}, 2 \mathrm{H}), 3.53(\mathrm{~s}, 3 \mathrm{H}), 1.86(\mathrm{~m}, 2 \mathrm{H}), 1.52(\mathrm{~m}, 2 \mathrm{H}) ;{ }^{13} \mathrm{C} \mathrm{NMR}\left(\mathrm{CDCl}_{3}, 100 \mathrm{MHz}\right) \delta 189.9,164.8$, $150.8,142.6,132.6,131.1,129.8,128.4,115.9,66.1,52.1,52.1,20.9,20.6$; IR $\left(\mathrm{NaCl}\right.$ plate, $\left.\mathrm{cm}^{-1}\right): 1714$, $1257,1201,1051$; Elemental analysis calculated for $\mathrm{C}_{16} \mathrm{H}_{16} \mathrm{O}_{4}$ : C $70.57 \% ; \mathrm{H} 5.92 \%$. Found : $\mathrm{C} 70.80 \% ; \mathrm{H}$ $6.02 \%$.

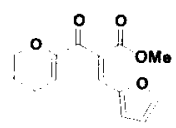

\section{2-(5,6-Dihydro-4H-pyran-2-carbonyl)-3-furan-2-yl-acrylic acid methyl ester}

3e ${ }^{1} \mathrm{H}$ NMR $\left(400 \mathrm{MHz}, \mathrm{CDCl}_{3}\right) \delta 7.34(\mathrm{~s}, 1 \mathrm{H}), 7.28(\mathrm{~d}, J=1.2 \mathrm{~Hz}, 1 \mathrm{H}), 6.51(\mathrm{~d}, J=3.6 \mathrm{~Hz}, 1 \mathrm{H}), 6.26(\mathrm{q}$, $J=1.6 \mathrm{~Hz}, 1 \mathrm{H}), 5.77(\mathrm{t}, J=3.6 \mathrm{~Hz}, 1 \mathrm{H}), 3.91(\mathrm{t}, J=4.8 \mathrm{~Hz}, 2 \mathrm{H}), 3.56(\mathrm{~s}, 3 \mathrm{H}), 1.98(\mathrm{~m}, 2 \mathrm{H}), 1.63(\mathrm{t}, J=$ $5.2 \mathrm{~Hz}, 2 \mathrm{H}) ;{ }^{13} \mathrm{C} \mathrm{NMR}\left(\mathrm{CDCl}_{3}, 100 \mathrm{MHz}\right) \delta 189.0,164.9,151.1,148.9,146.0,128.2,125.8,117.8,115.5$, 
112.4, 66.2, 52.1, 22.1, 20.7; IR ( NaCl plate, $\left.\mathrm{cm}^{-1}\right): 1710,1671,1626,1253,1212$; Elemental analysis calculated for $\mathrm{C}_{14} \mathrm{H}_{14} \mathrm{O}_{5}$ : C 64.12\%; $\mathrm{H} 5.38 \%$. Found : $\mathrm{C} 63.83 \%$; $\mathrm{H} 5.42 \%$.

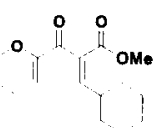

\section{3-Cyclohexyl-2-(5,6-dihydro-4H-pyran-2-carbonyl)-acrylic acid methyl ester}

3f ${ }^{1} \mathrm{H}$ NMR (400 MHz, $\left.\mathrm{CDCl}_{3}\right) \delta 6.69(\mathrm{~d}, J=10.8 \mathrm{~Hz}, 1 \mathrm{H}), 5.85(\mathrm{t}, J=4.4 \mathrm{~Hz}, 1 \mathrm{H}), 3.99(\mathrm{t}, J=5.2 \mathrm{~Hz}$, $2 \mathrm{H}), 3.60(\mathrm{~s}, 3 \mathrm{H}), 2.08(\mathrm{~m}, 2 \mathrm{H}), 2.03(\mathrm{~m}, 1 \mathrm{H}), 1.76(\mathrm{~m}, 2 \mathrm{H}), 1.54(\mathrm{~m}, 5 \mathrm{H}), 1.02(\mathrm{~m}, 5 \mathrm{H}) ;{ }^{13} \mathrm{C} \mathrm{NMR}\left(\mathrm{CDCl}_{3}\right.$, $100 \mathrm{MHz}) \delta 189.1,164.9,152.7,151.5,130.4,115.4,66.2,51.8,38.4,31.3,25.3,24.8,21.1,20.8$; IR $\left(\mathrm{NaCl}\right.$ plate, $\left.\mathrm{cm}^{-1}\right): 2925,2853,1738,1713,1645,1444,1300,1266,1216,1162,1123,1071,1034$; Elemental analysis calculated for $\mathrm{C}_{16} \mathrm{H}_{22} \mathrm{O}_{4}$ : C 69.04\%; $\mathrm{H} 7.97 \%$. Found : $\mathrm{C} 69.17 \% ; \mathrm{H} .79 \%$.

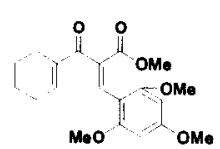

2-(Cyclohex-1-enecarbonyl)-3-(2,4,6-trimethoxy-phenyl)-acrylic acid methyl ester 5a ${ }^{\mathrm{I}} \mathrm{H}$ NMR $\left(400 \mathrm{MHz}, \mathrm{CDCl}_{3}\right) \delta 7.98(\mathrm{~s}, 1 \mathrm{H}), 6.62(\mathrm{~s}, 1 \mathrm{H}), 6.06(\mathrm{~s}, 2 \mathrm{H}), 3.78(\mathrm{~s}, 3 \mathrm{H}), 3.76(\mathrm{~s}, 3 \mathrm{H}), 3.71$ $(\mathrm{s}, 6 \mathrm{H}), 2.25(\mathrm{~m}, 2 \mathrm{H}), 2.04(\mathrm{~m}, 2 \mathrm{H}), 1.52(\mathrm{~m}, 4 \mathrm{H}) ;{ }^{13} \mathrm{C} \mathrm{NMR}\left(\mathrm{CDCl}_{3}, 100 \mathrm{MHz}\right) \delta 194.8,167.3,163.1$, $159.3,141.5,138.2,134.9,128.4,105.3,89.9,55.2,54.9,51.9,25.8,22.8,21.8,21.6$; IR (NaCl plate, $\left.\mathrm{cm}^{-1}\right)$ : $1710,1603,1251,1206,1157,1128$; Elemental analysis calculated for $\mathrm{C}_{20} \mathrm{H}_{24} \mathrm{O}_{6}: \mathrm{C} 66.65 \% ; \mathrm{H}, 6.71 \%$. Found : C $66.48 \%$; H $6.56 \%$.

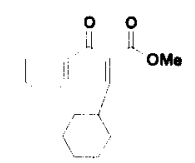

\section{2-(Cyclohex-1-enecarbonyl)-3-cyclohexyl-acrylic acid methyl ester}

5f A separable mixture of $E$ and $Z$ stereoisomers were obtained in a ratio of 10:1 (ratio of mass after column on silica). The geometry was assigned by comparing the chemical shift of the vinylic proton at $\beta$ ' position. E isomer: ' $\mathrm{H}$ NMR $\left(400 \mathrm{MHz}, \mathrm{CDCl}_{3}\right) \delta 6.63(\mathrm{~m}, 2 \mathrm{H}), 3.56(\mathrm{~s}, 3 \mathrm{H}), 2.10(\mathrm{~m}, 4 \mathrm{H}), 1.88(\mathrm{~m}, 1 \mathrm{H})$, $1.51(\mathrm{~m}, 9 \mathrm{H}), 1.02(\mathrm{~m}, 5 \mathrm{H}) ;{ }^{13} \mathrm{C} \mathrm{NMR}\left(\mathrm{CDCl}_{3}, 100 \mathrm{MHz}\right) \delta 195.3,165.1,150.8,144.6,139.9,131.2,51.7$, 38.5, 31.3, 25.9, 25.3, 24.8, 22.2, 21.5, 21.3; IR ( NaCl plate, $\left.\mathrm{cm}^{-1}\right): 2927,2852,1722,1658,1254,1228$; Elemental analysis calculated for $\mathrm{C}_{17} \mathrm{H}_{24} \mathrm{O}_{3}$ : C $73.88 \%$; $\mathrm{H} .75 \%$. Found : $\mathrm{C} 74.12 \% ; \mathrm{H} 8.54 \%$.

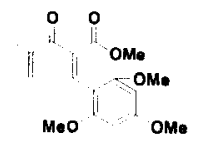

\section{4-Methyl-3-oxo-2-(2,4,6-trimethoxy-benzylidene)-pent-4-enoic acid methyl ester}

7a 'H NMR (400 MHz, CDCl $) \delta 7.95(\mathrm{~s}, 1 \mathrm{H}), 5.96(\mathrm{~s}, 2 \mathrm{H}), 5.71(\mathrm{~s}, 1 \mathrm{H}), 5.61(\mathrm{~s}, 1 \mathrm{H}), 3.76(\mathrm{~s}, 3 \mathrm{H}), 3.71$ $(\mathrm{s}, 3 \mathrm{H}), 3.65(\mathrm{~s}, 6 \mathrm{H}), 1.85(\mathrm{~s}, 3 \mathrm{H}) ;{ }^{13} \mathrm{C} \mathrm{NMR}\left(\mathrm{CDCl}_{3}, 100 \mathrm{MHz}\right) \delta 195.2,167.2,163.4,159.5,143.6,135.0$, $127.8,125.8,105.8,90.1,55.2,54.9,51.9,16.8$; IR (NaCl plate, $\left.\mathrm{cm}^{-1}\right): 1710,1664,1603,1462,1436,1252$, 
$1208,1158,1129,735$; Elemental analysis calculated for $\mathrm{C}_{17} \mathrm{H}_{20} \mathrm{O}_{6}: \mathrm{C} 63.74 \% ; \mathrm{H} 6.29 \%$. Found : C 63.86\%; H $6.35 \%$.

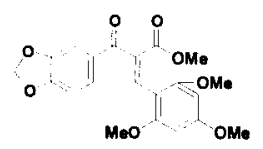

2-(Benzo[1,3]dioxole-5-carbonyl)-3-(2,4,6-trimethoxy-phenyl)-acrylic acid methyl ester 9a ${ }^{1} \mathrm{H}$ NMR $\left(400 \mathrm{MHz}, \mathrm{CDCl}_{3}\right) \delta 8.15(\mathrm{~s}, 1 \mathrm{H}), 7.48(\mathrm{q}, J=9.8 \mathrm{~Hz}, 1 \mathrm{H}), 7.44(\mathrm{~d}, J=1.6 \mathrm{~Hz}, 1 \mathrm{H}), 6.79(\mathrm{~d}$, $J=8.1 \mathrm{~Hz}, 1 \mathrm{H}), 6.03(\mathrm{~s}, 2 \mathrm{H}), 5.96(\mathrm{~s}, 2 \mathrm{H}), 3.79(\mathrm{~s}, 3 \mathrm{H}), 3.72(\mathrm{~s}, 3 \mathrm{H}), 3.53(\mathrm{~s}, 6 \mathrm{H}) ;{ }^{13} \mathrm{C} \mathrm{NMR}\left(\mathrm{CDCl}_{3}, 100\right.$ $\mathrm{MHz}) \delta 191.8167 .2,163.4,159.6,151.0,147.6,135.5,132.5,128.0,125.1,108.2,107.5,105.0,101.5$, 90.7, 55.2, 54.6, 52.0; IR (NaCl plate, $\mathrm{cm}^{-1}$ ): 1707, 1664, 1603, 1439, 1254, 1209, 1158, 1129, 1035; Elemental analysis calculated for $\mathrm{C}_{21} \mathrm{H}_{20} \mathrm{O}_{8}: \mathrm{C} 63.00 \% ; \mathrm{H} 5.03 \%$. Found : $\mathrm{C} 63.24 \% ; \mathrm{H} 4.99 \%$.

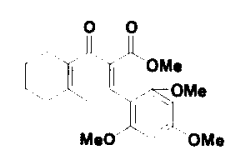

2-(2-Methyl-cyclohex-1-enecarbonyl)-3-(2,4,6-trimethoxy-phenyl)-acrylic acid methyl ester $11 \mathrm{a}{ }^{\prime} \mathrm{H}$ NMR $\left(400 \mathrm{MHz}, \mathrm{CDCl}_{3}\right) \delta 7.72(\mathrm{~s}, 1 \mathrm{H}), 6.00(\mathrm{~s}, 2 \mathrm{H}), 3.74(\mathrm{~s}, 3 \mathrm{H}), 3.71(\mathrm{~s}, 3 \mathrm{H}), 3.63(\mathrm{~s}, 6 \mathrm{H}), 2.11$ (m, 2H), $2.00(\mathrm{~m}, 2 \mathrm{H}), 1.88(\mathrm{~s}, 3 \mathrm{H}), 1.43(\mathrm{~m}, 4 \mathrm{H}) ;{ }^{13} \mathrm{C} \mathrm{NMR}\left(\mathrm{CDCl}_{3}, 100 \mathrm{MHz}\right) \delta 195.5,166.9,162.9$, $159.1,145.7,134.6,132.5,131.8,105.1,90.2,55.3,55.2,51.8,34.2,26.5,22.5,22.3,22.0 ; \mathrm{IR}$ (NaCl plate, $\mathrm{cm}^{-1}$ ): $2935,1726,1602,1459,1336,1232,1208,1157,1129$; Elemental analysis calculated for $\mathrm{C}_{21} \mathrm{H}_{26} \mathrm{O}_{6}$ : C $67.36 \%$; H $7.00 \%$. Found : C $67.50 \%$; H $6.98 \%$.

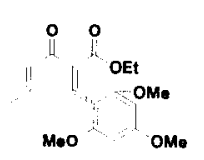

\section{3-Oxo-2-(2,4,6-trimethoxy-benzylidene)-hex-4-enoic acid ethyl ester}

13a As an unseparable mixture of $Z$ and $E$ stereoisomers (0.6: 1.0 according to the integration on ${ }^{1} \mathrm{H}$ NMR ). ${ }^{\mathrm{H}} \mathrm{H}$ NMR $\left(400 \mathrm{MHz}, \mathrm{CDCl}_{3}\right) \delta 7.95(\mathrm{~s}, 1 \mathrm{H}), 7.78(\mathrm{~s}, 0.6 \mathrm{H}), 7.00(\mathrm{~m}, 0.6 \mathrm{H}), 6.68(\mathrm{~m}, 1 \mathrm{H}), 6.19(\mathrm{~d}, J$ $=1.6 \mathrm{~Hz}, 0.6 \mathrm{H}), 6.15(\mathrm{~d}, J=1.6 \mathrm{~Hz}, 1 \mathrm{H}), 6.08(\mathrm{~s}, 1.2 \mathrm{H}), 6.04(\mathrm{~s}, 2 \mathrm{H}), 4.24(\mathrm{~m}, 2 \mathrm{H}), 4.13(\mathrm{~m}, 1.2 \mathrm{H}), 3.88(\mathrm{~s}$, $2.4 \mathrm{H}), 3.83(\mathrm{~s}, 3.6 \mathrm{H}), 3.79(\mathrm{~s}, 6 \mathrm{H}), 3.73(\mathrm{~s}, 6 \mathrm{H}), 1.92(\mathrm{~d}, J=1.6 \mathrm{~Hz}, 1.2 \mathrm{H}), 1.82(\mathrm{~d}, J=1.8 \mathrm{~Hz}, 2 \mathrm{H}), 1.28$ $(\mathrm{m}, 1.8 \mathrm{H}), 1.24(\mathrm{~m}, 3 \mathrm{H}) ;{ }^{13} \mathrm{C}$ NMR $\left(\mathrm{CDCl}_{3}, 100 \mathrm{MHz}\right) \delta 193.0,188.3,167.8,166.5,163.5,163.3,159.8$, $159.5,143.7,142.4,136.1,135.1,133.0,132.0,129.6,128.6,105.8,105.2,90.2,60.9,60.4,55.4,55.3$, $54.9,18.4,18.1,14.2,13.9$; IR ( NaCl plate, $\left.\mathrm{cm}^{-1}\right): 1653,1605,1207,1155,1128,500$; Elemental analysis calculated for $\mathrm{C}_{18} \mathrm{H}_{22} \mathrm{O}_{6}$ : C $64.66 \% ; \mathrm{H} 6.63 \%$. Found : $\mathrm{C} 64.75 \% ; \mathrm{H} 6.52 \%$.

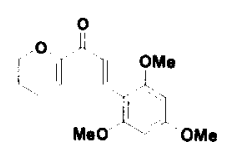

1-(5,6-Dihydro-4H-pyran-2-yl)-3-(2,4,6-trimethoxy-phenyl)-propenone

15a 'H NMR $\left(400 \mathrm{MHz}, \mathrm{CDCl}_{3}\right) \delta 8.14(\mathrm{~d}, J=16 \mathrm{~Hz}, 1 \mathrm{H}), 7.58(\mathrm{~d}, J=16 \mathrm{~Hz}, 1 \mathrm{H}), 6.08(\mathrm{~s}, 2 \mathrm{H}), 6.02(\mathrm{t}$, 
$J=3.6 \mathrm{~Hz}, 1 \mathrm{H}), 4.11(\mathrm{~m}, 2 \mathrm{H}), 3.85(\mathrm{~s}, 6 \mathrm{H}), 3.82(\mathrm{~s}, 3 \mathrm{H}), 2.23(\mathrm{~m}, 2 \mathrm{H}), 1.86(\mathrm{~m}, 2 \mathrm{H}) ;{ }^{13} \mathrm{C} \mathrm{NMR}\left(\mathrm{CDCl}_{3}\right.$, $100 \mathrm{MHz}) \delta 186.7,162.8,161.5,152.3,134.8,120.0,109.6,106.3,90.2,66.1,55.6,55.2,21.5,20.8$; IR $\left(\mathrm{NaCl}\right.$ plate, $\left.\mathrm{cm}^{-1}\right): 1567,1463,1324,1206,1177,1157,1120,1062$; Elemental analysis calculated for $\mathrm{C}_{17} \mathrm{H}_{20} \mathrm{O}_{5}$ : C 67.09\%; H 6.62\%. Found : C 67.18\%; H 6.69\%.

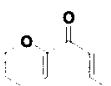

\section{3-Cyclohexyl-1-(5,6-dihydro-4H-pyran-2-yl)-propenone}

$15 f{ }^{\prime} \mathrm{H} \mathrm{NMR}\left(400 \mathrm{MHz}, \mathrm{CDCl}_{3}\right) \delta 6.96\left(\mathrm{dd}, J_{1}=15.6 \mathrm{~Hz}, J_{2}=6.8 \mathrm{~Hz}, 1 \mathrm{H}\right), 6.62(\mathrm{~d}, J=15.6 \mathrm{~Hz}, 1 \mathrm{H})$, $6.02(\mathrm{t}, J=8 \mathrm{~Hz}, 1 \mathrm{H}), 4.11(\mathrm{~m}, 2 \mathrm{H}), 2.24(\mathrm{~m}, 2 \mathrm{H}), 2.21(\mathrm{~m}, 1 \mathrm{H}), 1.86(\mathrm{~m}, 2 \mathrm{H}), 1.17-1.75(\mathrm{~m}, 10 \mathrm{H}) ;{ }^{13} \mathrm{C}$ NMR $\left(\mathrm{CDCl}_{3}, 100 \mathrm{MHz}\right) \delta 185.9,153.8,151.7,121.4,110.7,66.25,43.3,31.7,26.4,26.2,21.5,20.8$; IR ( $\mathrm{NaCl}$ plate, $\mathrm{cm}^{-1}$ ): 2926, 2853, 1725, 1632, 1448, 1309, 1280, 1243, 1176, 1062, 916; Elemental analysis calculated for $\mathrm{C}_{14} \mathrm{H}_{20} \mathrm{O}_{2}$ : C 76.33\%; $\mathrm{H} 9.15 \%$. Found : C $76.18 \%$; $9.19 \%$.

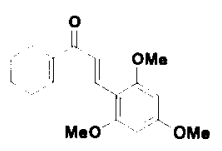

\section{1-Cyclohex-1-enyl-3-(2,4,6-trimethoxy-phenyl)-propenone}

$17 \mathrm{a}{ }^{1} \mathrm{H}$ NMR $\left(400 \mathrm{MHz}, \mathrm{CDCl}_{3}\right) \delta 8.02(\mathrm{~d}, J=16 \mathrm{~Hz}, 1 \mathrm{H}), 7.60(\mathrm{~d}, J=16 \mathrm{~Hz}, 1 \mathrm{H}), 6.91(\mathrm{t}, J=4 \mathrm{~Hz}$, $1 \mathrm{H}), 6.09(\mathrm{~s}, 2 \mathrm{H}), 3.85(\mathrm{~s}, 6 \mathrm{H}), 3.81(\mathrm{~s}, 3 \mathrm{H}), 2.34(\mathrm{~m}, 2 \mathrm{H}), 2.26(\mathrm{~m}, 2 \mathrm{H}), 1.64(\mathrm{~m}, 4 \mathrm{H}) ;{ }^{13} \mathrm{C} \mathrm{NMR}\left(\mathrm{CDCl}_{3}\right.$, $100 \mathrm{MHz}) \delta 192.7,162.4,161.2,140.3,138.1,133.5,121.4,106.5,90.3,55.6,55.2,26.0,23.6,22.0,21.6$; IR $\left(\mathrm{NaCl}\right.$ plate, $\left.\mathrm{cm}^{-1}\right): 2933,1642,1571,1458,1322,1205,1156,1120$; Elemental analysis calculated for $\mathrm{C}_{18} \mathrm{H}_{22} \mathrm{O}_{4}$ : C 71.50\%; $\mathrm{H} 7.33 \%$. Found : C 71.68\%; H 7.25\%.

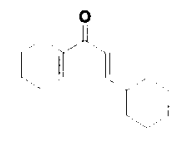

\section{1-Cyclohex-1-enyl-3-cyclohexyl-propenone}

17f ' $\mathrm{H}$ NMR $\left(400 \mathrm{MHz}, \mathrm{CDCl}_{3}\right) \delta 6.84$ (br. m, $\left.1 \mathrm{H}\right), 6.74\left(\mathrm{dd}, J_{1}=15.2 \mathrm{~Hz}, J_{2}=7.8 \mathrm{~Hz}, 1 \mathrm{H}\right), 6.52(\mathrm{~d}, J=$ $15.2 \mathrm{~Hz}, 1 \mathrm{H}), 2.20(\mathrm{~m}, 4 \mathrm{H}), 2.08(\mathrm{~m}, 1 \mathrm{H}), 1.70(\mathrm{~m}, 4 \mathrm{H}), 1.56(\mathrm{~m}, 5 \mathrm{H}), 1.25(\mathrm{~m}, 2 \mathrm{H}), 1.15(\mathrm{~m}, 3 \mathrm{H}) ;{ }^{13} \mathrm{C}$ $\mathrm{NMR}\left(\mathrm{CDCl}_{3}, 100 \mathrm{MHz}\right) \delta 191.4,151.6,139.7,139.4,122.0,40.6,31.8,25.9,25.8,25.6,25.6,23.2,21.8$, 21.4; IR ( $\mathrm{NaCl}$ plate, $\left.\mathrm{cm}^{-1}\right): 2924,2852,1659,1613$; Elemental analysis calculated for $\mathrm{C}_{15} \mathrm{H}_{22} \mathrm{O}: \mathrm{C} 82.52 \%$; H $10.16 \%$. Found : C $82.19 \%$; H $9.90 \%$.

\section{Representive procedure of $\mathrm{Cu}(\mathrm{OTf})_{2}$ catalyzed Nazarov cyclization}

To a stirred suspension of $\mathrm{Cu}(\mathrm{OTf})_{2}(2.2 \mathrm{mg}, 0.006 \mathrm{mmol}, 0.02 \mathrm{eq}$. $)$ in dichloroethane $(0.5 \mathrm{~mL})$ was added a solution of $5 \mathrm{a}(110 \mathrm{mg}, 0.304 \mathrm{mmol}, 1.00 \mathrm{eq}$.) in dichloroethane $(1 \mathrm{~mL})$. The resulting mixture was heated to $40{ }^{\circ} \mathrm{C}$ under vigirous stirring. After 15 minutes, TLC found complete consumption of the starting material. The reaction was then cooled down to room temperature and purified by flash chromatography on 
silica eluting with 20\% EtOAc/Hexanes to give 6a-1( $43 \mathrm{mg} \mathrm{40 \% )} \mathrm{and} \mathrm{6a-2} \mathrm{(49} \mathrm{mg}, 45 \%$ ).

\section{Spectroscopic data of Nazarov products}

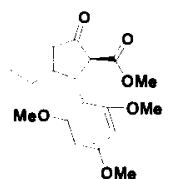

methyl ester

6a-1 'H NMR (400 MHz, $\left.\mathrm{CDCl}_{3}\right) \delta 6.80\left(\mathrm{dd}, J_{1}=6.4 \mathrm{~Hz}, J_{2}=3.2 \mathrm{~Hz}, 1 \mathrm{H}\right), 6.14(\mathrm{~s}, 2 \mathrm{H}), 4.30(\mathrm{~d}, J=$ $12.1 \mathrm{~Hz}, 1 \mathrm{H}), 3.80(\mathrm{t}, J=12.1 \mathrm{~Hz}, 1 \mathrm{H}), 3.77(\mathrm{~s}, 3 \mathrm{H}), 3.74(\mathrm{~s}, 6 \mathrm{H}), 3.65(\mathrm{~s}, 3 \mathrm{H}), 2.93(\mathrm{~m}, 1 \mathrm{H}), 2.04(\mathrm{~m}, 2 \mathrm{H})$, $1.80(\mathrm{~m}, 2 \mathrm{H}), 1.25(\mathrm{~m}, 1 \mathrm{H}), 1.13(\mathrm{~m}, 1 \mathrm{H}) ;{ }^{13} \mathrm{C} \mathrm{NMR}\left(\mathrm{CDCl}_{3}, 100 \mathrm{MHz}\right) \delta 199.4,170.3,159.9,159.7,140.6$, $134.4,107.5,91.1,91.0,57.6,55.2,55.0,52.0,40.4,39.6,27.1,25.6,21.5 ; \mathrm{IR}\left(\mathrm{NaCl}\right.$ plate, $\left.\mathrm{cm}^{-1}\right): 1742$, $1711,1606,1459,1202,1151,1115$; Elemental analysis calculated for $\mathrm{C}_{20} \mathrm{H}_{24} \mathrm{O}_{6}: \mathrm{C} 66.65 \% ; \mathrm{H}, 6.71 \%$. Found : C $66.80 \%$; H $6.75 \%$.

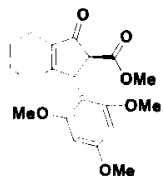

1-Ox0-3-(2,4,6-trimethoxy-phenyl)-2,3,4,5,6,7-hexahydro-1H-indene-2-carboxylic acid methyl ester

6a-2 'H NMR (400 MHz, $\left.\mathrm{CDCl}_{3}\right) \delta 6.16(\mathrm{~d}, J=2.4 \mathrm{~Hz}, 1 \mathrm{H}), 6.06(\mathrm{~d}, J=2.4 \mathrm{~Hz}, 1 \mathrm{H}), 4.84(\mathrm{~m}, 1 \mathrm{H}), 3.81$ (s, 6H), $3.74(\mathrm{~s}, 3 \mathrm{H}), 3.67(\mathrm{~s}, 3 \mathrm{H}), 3.59(\mathrm{~d}, J=5.8 \mathrm{~Hz}, 1 \mathrm{H}), 2.00(\mathrm{~m}, 2 \mathrm{H}), 1.73(\mathrm{~m}, 1 \mathrm{H}), 1.71(\mathrm{~m}, 1 \mathrm{H}), 1.63$ $(\mathrm{m}, 4 \mathrm{H}) ;{ }^{13} \mathrm{C} \mathrm{NMR}\left(\mathrm{CDCl}_{3}, 100 \mathrm{MHz}\right) \delta 201.6,177.1,170.7,160.4,159.4,159.2,134.9,107.2,90.7,58.2$, 56.0, 55.2, 55.1, 41.6, 26.2, 22.1, 21.5, 20.2; IR (NaCl plate, $\left.\mathrm{cm}^{-1}\right): 2937,1737,1697,1643,1604,1459$, $1436,1332,1258,1228,1205,1150,1116,733$; Elemental analysis calculated for $\mathrm{C}_{20} \mathrm{H}_{24} \mathrm{O}_{6}$ : $\mathrm{C} 66.65 \% ; \mathrm{H}$, $6.71 \%$. Found : C $66.81 \%$; H $6.62 \%$.

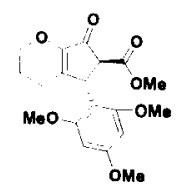

7-Oxo-5-(2,4,6-trimethoxy-phenyl)-2,3,4,5,6,7-hexahydro-cyclopenta[b]pyran-6-carboxylic acid methyl ester

4a ${ }^{1} \mathrm{H} \mathrm{NMR}\left(400 \mathrm{MHz}, \mathrm{CDCl}_{3}\right) \delta 6.11(\mathrm{~s}, 1 \mathrm{H}), 6.04(\mathrm{~s}, 1 \mathrm{H}), 4.78(\mathrm{~d}, J=1.6 \mathrm{~Hz}, 1 \mathrm{H}), 4.05(\mathrm{t}, J=4.6 \mathrm{~Hz}$, 2H), $3.74(\mathrm{~s}, 6 \mathrm{H}), 3.69(\mathrm{~s}, 3 \mathrm{H}), 3.64(\mathrm{~s}, 3 \mathrm{H}), 3.54(\mathrm{~d}, J=1.6 \mathrm{~Hz}, 1 \mathrm{H}), 1.92(\mathrm{~m}, 1 \mathrm{H}), 1.90(\mathrm{~m}, 1 \mathrm{H}), 1.87(\mathrm{~m}$, $1 \mathrm{H}), 1.82(\mathrm{~m}, 1 \mathrm{H}) ;{ }^{13} \mathrm{C} \mathrm{NMR}\left(\mathrm{CDCl}_{3}, 100 \mathrm{MHz}\right) \delta 193.9,170.0,160.5,149.5,148.2,106.2,90.7,66.6$, $56.1,55.9,52.2,37.1,22.0,21.3$; IR $\left(\mathrm{NaCl}\right.$ plate $\left.\mathrm{cm}^{-1}\right): 1738,1708,1605,1205,1150,1120 ; \mathrm{X}$-ray crystallography please see attached cif file. 


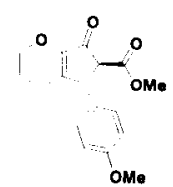

5-(4-Methoxy-phenyl)-7-oxo-2,3,4,5,6,7-hexahydro-cyclopenta[b]pyran-6-carboxylic acid methyl ester

4b ${ }^{~} \mathrm{H}$ NMR (400 MHz, $\mathrm{CDCl}_{3}$ ) $\delta 7.01(\mathrm{~d}, J=8.8 \mathrm{~Hz}, 2 \mathrm{H}), 6.83(\mathrm{~d}, J=8.8 \mathrm{~Hz}, 2 \mathrm{H}), 4.13(\mathrm{~m}, 3 \mathrm{H}), 3.73$ (s, $3 \mathrm{H}), 3.71(\mathrm{~s}, 3 \mathrm{H}), 3.24(\mathrm{~d}, J=2.4 \mathrm{~Hz}, 1 \mathrm{H}), 2.10(\mathrm{~m}, 2 \mathrm{H}), 1.90(\mathrm{~m}, 2 \mathrm{H}) ;{ }^{13} \mathrm{C} \mathrm{NMR}\left(\mathrm{CDCl}_{3}, 100 \mathrm{MHz}\right) \delta$ $193.0,168.7,158.9,149.5,147.8,131.5,128.2,114.4,66.9,59.3,55.2,52.6,46.8,22.0,22.1 ; \mathrm{IR}(\mathrm{NaCl}$ plate, $\left.\mathrm{cm}^{-1}\right): 1738,1713,1648,1512,1250,1162,1122$; HRMS calculated for $\mathrm{C}_{19} \mathrm{H}_{22} \mathrm{O}_{7}(\mathrm{M}+\mathrm{H})^{+} 303.1216$, Found : 303.1227 .

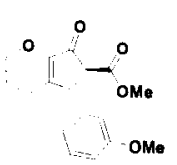

5-(3-Methoxy-phenyl)-7-oxo-2,3,4,5,6,7-hexahydro-cyclopenta[b]pyran-6-carboxylic acid methyl ester

4c ' $\mathrm{H}$ NMR $\left(400 \mathrm{MHz}, \mathrm{CDCl}_{3}\right) \delta 7.23(\mathrm{t}, J=8 \mathrm{~Hz}, 1 \mathrm{H}), 6.78\left(\mathrm{dd}, J_{1}=8 \mathrm{~Hz}, J_{2}=6 \mathrm{~Hz}, 1 \mathrm{H}\right), 6.69(\mathrm{~d}, J=$ $7.6 \mathrm{~Hz}, 1 \mathrm{H}), 6.64(\mathrm{~d}, J=1.6 \mathrm{~Hz}, 1 \mathrm{H}), 4.16$ (br. s, $1 \mathrm{H}), 4.08(\mathrm{~m}, 2 \mathrm{H}), 3.75(\mathrm{~s}, 3 \mathrm{H}), 3.73(\mathrm{~s}, 3 \mathrm{H}), 3.29$ (br. s, $1 \mathrm{H}), 2.10(\mathrm{~m}, 2 \mathrm{H}), 1.88(\mathrm{~m}, 2 \mathrm{H}) ;{ }^{13} \mathrm{C} \mathrm{NMR}\left(\mathrm{CDCl}_{3}, 100 \mathrm{MHz}\right) \delta 192.8,168.6,160.0,149.7,147.3,141.3$, 130.1, 119.4, 113.2, 112.6, 66.9, 58.9, 58.9, 55.1, 47.5, 22.1, 21.1; IR (NaCl plate, $\left.\mathrm{cm}^{-1}\right)$ : 1736, 1714, 439.3; Elemental analysis calculated for $\mathrm{C}_{17} \mathrm{H}_{18} \mathrm{O}_{5}$ : $\mathrm{C} 67.54 \% ; \mathrm{H} \mathrm{6.00 \%}$. Found : $\mathrm{C} 67.62 \% ; \mathrm{H} 5.94 \%$.

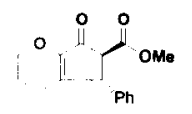

7-Oxo-5-phenyl-2,3,4,5,6,7-hexahydro-cyclopenta[b]pyran-6-carboxylic acid methyl ester 4d 'H NMR $\left(400 \mathrm{MHz}, \mathrm{CDCl}_{3}\right) \delta 7.33(\mathrm{t}, J=7.2 \mathrm{~Hz}, 2 \mathrm{H}), 7.28(\mathrm{~d}, J=7.2 \mathrm{~Hz}, 1 \mathrm{H}), 7.13(\mathrm{~d}, J=7.2 \mathrm{~Hz}$, 2H), 4.20 (br. s, 1H), 4.15 (m, 2H), 3.75 (s, 3H), 3.32 (br. s, 1H), $2.13(\mathrm{~m}, 1 \mathrm{H}), 2.08(\mathrm{~m}, 1 \mathrm{H}), 1.93(\mathrm{~m}, 2 \mathrm{H})$; ${ }^{13} \mathrm{C} \mathrm{NMR}\left(\mathrm{CDCl}_{3}, 100 \mathrm{MHz}\right) \delta 192.8,168.6,149.7,147.4,139.7,128.8,127.6,127.2,66.9,59.1,52.8$, 47.5, 22.1, 21.1; IR (NaCl plate, $\left.\mathrm{cm}^{-1}\right): 1739,1714,1648,1263,1161,1123$; Elemental analysis calculated for $\mathrm{C}_{16} \mathrm{H}_{16} \mathrm{O}_{4}$ : C $70.57 \%$; $\mathrm{H} .92 \%$. Found : C $70.70 \%$; $5.78 \%$.

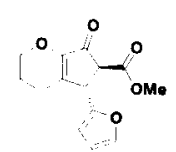

\section{5-Furan-2-yl-7-oxo-2,3,4,5,6,7-hexahydro-cyclopenta[b]pyran-6-carboxylic acid methyl ester}

4e ${ }^{1} \mathrm{H}$ NMR $\left(400 \mathrm{MHz}, \mathrm{CDCl}_{3}\right) \delta 7.33(\mathrm{~d}, J=1.2 \mathrm{~Hz}, 1 \mathrm{H}), 6.31(\mathrm{t}, J=1.2 \mathrm{~Hz}, 1 \mathrm{H}), 6.17(\mathrm{~d}, J=3.2 \mathrm{~Hz}$, $1 \mathrm{H}), 4.35$ (d, $J=2 \mathrm{~Hz}, 1 \mathrm{H}), 4.13(\mathrm{~m}, 2 \mathrm{H}), 3.76(\mathrm{~s}, 3 \mathrm{H}), 3.52(\mathrm{~d}, J=2 \mathrm{~Hz}, 1 \mathrm{H}), 2.27(\mathrm{~m}, 1 \mathrm{H}), 2.19(\mathrm{~m}, 1 \mathrm{H})$, $1.94(\mathrm{~m}, 2 \mathrm{H}) ;{ }^{13} \mathrm{C} \mathrm{NMR}\left(\mathrm{CDCl}_{3}, 100 \mathrm{MHz}\right) \delta 192.0,168.4,151.7,149.4,144.7,142.4,110.3,107.2,66.9$, $55.5,52.8,40.8,22.2,21.1$; IR ( NaCl plate, $\left.\mathrm{cm}^{-1}\right): 1715,1649,524$; Elemental analysis calculated for $\mathrm{C}_{14} \mathrm{H}_{14} \mathrm{O}_{5}$ : C $64.12 \%$; H 5.38\%. Found : C 64.00\%; H 5.51\%. 


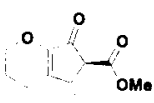

5-Cyclohexyl-7-oxo-2,3,4,5,6,7-hexahydro-cyclopenta[b]pyran-6-carboxylic acid methyl ester 4f ${ }^{\prime} \mathrm{H}$ NMR $\left(400 \mathrm{MHz}, \mathrm{CDCl}_{3}\right) \delta 4.16(\mathrm{~m}, 1 \mathrm{H}), 4.03(\mathrm{~m}, 1 \mathrm{H}), 3.72(\mathrm{~s}, 3 \mathrm{H}), 3.17(\mathrm{~d}, J=2.4 \mathrm{~Hz}, 1 \mathrm{H}), 3.03$ $(\mathrm{d}, J=2.4 \mathrm{~Hz}, 1 \mathrm{H}), 2.33(\mathrm{~m}, 2 \mathrm{H}), 1.93(\mathrm{~m}, 2 \mathrm{H}), 1.69-1.74(\mathrm{~m}, 4 \mathrm{H}), 1.07-1.24(\mathrm{~m}, 7 \mathrm{H}) ;{ }^{13} \mathrm{C} \mathrm{NMR}\left(\mathrm{CDCl}_{3}\right.$, $100 \mathrm{MHz}) \delta 194.1,170.0,149.1,147.8,66.8,52.6,51.5,47.4,38.2,31.0,26.6,26.3,26.1,26.0,22.7,21.2$; IR $\left(\mathrm{NaCl}\right.$ plate, $\left.\mathrm{cm}^{-1}\right): 2359,2337,1711,1033,824,614$; Elemental analysis calculated for $\mathrm{C}_{16} \mathrm{H}_{22} \mathrm{O}_{4}: \mathrm{C}$ $69.04 \%$; H 7.97\%. Found : C $68.84 \%$; H 7.93\%.

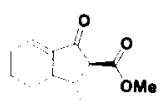

3-Cyclohexyl-1-oxo-2,3,3a,4,5,6-hexahydro-1H-indene-2-carboxylic acid methyl ester 6f-1 $\left(53 \%\right.$ isolated yield) ${ }^{1} \mathrm{H}$ NMR $\left(400 \mathrm{MHz}, \mathrm{CDCl}_{3}\right) \delta 6.79(\mathrm{t}, J=3.6 \mathrm{~Hz}, 1 \mathrm{H}), 3.76(\mathrm{~s}, 3 \mathrm{H}), 3.16(\mathrm{~d}, J=$ 11.6, $1 \mathrm{H}), 2.36(\mathrm{~m}, 2 \mathrm{H}), 2.20(\mathrm{~m}, 2 \mathrm{H}), 2.12(\mathrm{~m}, 1 \mathrm{H}), 1.76(\mathrm{~m}, 1 \mathrm{H}), 1.65(\mathrm{~m}, 5 \mathrm{H}), 1.55(\mathrm{~m}, 2 \mathrm{H}), 1.15(\mathrm{~m}, 6 \mathrm{H})$; ${ }^{13} \mathrm{C} \mathrm{NMR}\left(\mathrm{CDCl}_{3}, 100 \mathrm{MHz}\right) \delta 199.2,171.2,140.2,135.4,57.8,52.4,51.1,39.7,38.7,31.7,29.3,28.4$, 26.7, 26.5, 26.3, 25.6, 21.7; IR ( NaCl plate, $\left.\mathrm{cm}^{-1}\right): 2925,2853,1744,1713,1651,1447,1336,1259,1149$, 979, 422; Elemental analysis calculated for $\mathrm{C}_{17} \mathrm{H}_{24} \mathrm{O}_{3}$ : C 73.88\%; $\mathrm{H} 8.75 \%$. Found : $\mathrm{C} 73.98 \%$; $\mathrm{H} 8.69 \%$.

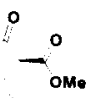

1-Cyclohexyl-3-oxo-2,3,4,5,6,7-hexahydro-1H-indene-2-carboxylic acid methyl ester 6f-2 (17\% isolated yield) ' $\mathrm{H}$ NMR $\left(400 \mathrm{MHz}, \mathrm{CDCl}_{3}\right) \delta 3.75(\mathrm{~s}, 3 \mathrm{H}), 3.22(\mathrm{~d}, J=2.4 \mathrm{~Hz}, 1 \mathrm{H}), 3.11$ (br. s, $1 \mathrm{H}), 2.29(\mathrm{~m}, 2 \mathrm{H}), 2.12(\mathrm{~m}, 2 \mathrm{H}), 1.71(\mathrm{~m}, 9 \mathrm{H}), 1.26(\mathrm{~m}, 5 \mathrm{H}), 0.83(\mathrm{~m}, 1 \mathrm{H}) ;{ }^{13} \mathrm{C} \mathrm{NMR}\left(\mathrm{CDCl}_{3}, 100 \mathrm{MHz}\right) \delta$ $200.9,175.6,170.5,137.6,53.3,52.3,51.8,37.9,31.5,26.7,26.4,26.2,26.1,26.0,22.0,21.4,20.1$; IR $\left(\mathrm{NaCl}\right.$ plate, $\left.\mathrm{cm}^{-1}\right) 2925,2853,1738,1702,1644,1439,1263,1155,897,730$; Elemental analysis calculated for $\mathrm{C}_{17} \mathrm{H}_{24} \mathrm{O}_{3}$ : C 73.88\%; H 8.75\%. Found : C 74.07\%; H 8.60\%.

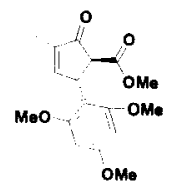

3-Methyl-2-oxo-5-(2,4,6-trimethoxy-phenyl)-cyclopent-3-enecarboxylic acid methyl ester 8a ${ }^{1} \mathrm{H}$ NMR $\left(400 \mathrm{MHz}, \mathrm{CDCl}_{3}\right) \delta 7.20(\mathrm{t}, J=1.2 \mathrm{~Hz}, 1 \mathrm{H}), 6.08(\mathrm{~s}, 2 \mathrm{H}), 4.90(\mathrm{q}, J=2.4 \mathrm{~Hz}, 1 \mathrm{H}), 3.76(\mathrm{~s}$, $3 \mathrm{H}), 3.73(\mathrm{~s}, 3 \mathrm{H}), 3.71(\mathrm{~s}, 6 \mathrm{H}), 3.63(\mathrm{~d}, J=2.4 \mathrm{~Hz}, 1 \mathrm{H}), 1.80(\mathrm{~s}, 3 \mathrm{H}) ;{ }^{1.3} \mathrm{C} \mathrm{NMR}\left(\mathrm{CDCl}_{3}, 100 \mathrm{MHz}\right) \delta 203.6$, $170.2,161.6,160.4,159.1,137.3,107.2,90.7,57.4,55.5,55.2,52.2,38.4,9.9 ; \mathrm{IR}\left(\mathrm{NaCl}\right.$ plate, $\left.\mathrm{cm}^{-1}\right): 1738$, $1704,1636,1605,1459,1436,1333,1256,1221,1204,1151,1119$; Elemental analysis calculated for $\mathrm{C}_{17} \mathrm{H}_{20} \mathrm{O}_{6}$ : C $63.74 \%$; $\mathrm{H} 6.29 \%$. Found : C $63.57 \%$; $6.13 \%$ 


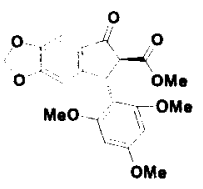

5-Ox0-7-(2,4,6-trimethoxy-phenyl)-6,7-dihydro-5H-indeno[5,6-d][1,3]dioxole-6-carboxylic acid methyl ester

10a ${ }^{1} \mathrm{H}$ NMR $\left(400 \mathrm{MHz}, \mathrm{CDCl}_{3}\right) \delta 7.11(\mathrm{~s}, 1 \mathrm{H}), 6.55(\mathrm{~s}, 1 \mathrm{H}), 6.21(\mathrm{~s}, 1 \mathrm{H}), 6.03(\mathrm{~s}, 3 \mathrm{H}), 5.42(\mathrm{~d}, J=3.6$ $\mathrm{Hz}, 1 \mathrm{H}), 4.00(\mathrm{~d}, J=3.6 \mathrm{~Hz}, 1 \mathrm{H}), 3.89(\mathrm{~s}, 3 \mathrm{H}), 3.82(\mathrm{~s}, 3 \mathrm{H}), 3.77(\mathrm{~s}, 3 \mathrm{H}), 3.47(\mathrm{~s}, 3 \mathrm{H}) ;{ }^{13} \mathrm{C} \mathrm{NMR}\left(\mathrm{CDCl}_{3}\right.$, $100 \mathrm{MHz}) \delta 197.9,170.2,160.5,156.2,154.3,147.9,129.0,109.0,104.4,102.0,91.1,90.6,59.8,56.0$, 55.2, 55.1, 52.3, 37.6; IR ( NaCl plate, $\mathrm{cm}^{-1}$ ): 1738, 1700, 1607, 1497, 1467, 1329, 1294, 1248, 1205, 1151, $1118,1035,815,734$; Elemental analysis calculated for $\mathrm{C}_{21} \mathrm{H}_{20} \mathrm{O}_{8}: \mathrm{C} 63.00 \% ; \mathrm{H} \mathrm{5.03 \%}$. Found : $\mathrm{C} 62.99 \%$; H $5.22 \%$.

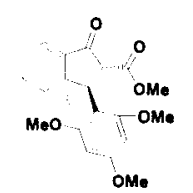

3a-Methyl-1-oxo-3-(2,4,6-trimethoxy-phenyl)-2,3,3a,4,5,6-hexahydro-1H-indene-2-carboxylic acid methyl ester

12a ${ }^{1} \mathrm{H}$ NMR $\left(400 \mathrm{MHz}, \mathrm{CDCl}_{3}\right) \delta 6.74(\mathrm{t}, J=4 \mathrm{~Hz}, 1 \mathrm{H}), 6.15(\mathrm{~s}, 2 \mathrm{H}), 4.90(\mathrm{~d}, J=13.2 \mathrm{~Hz}, 1 \mathrm{H}), 4.05(\mathrm{~d}$, $J=13.2 \mathrm{~Hz}, 1 \mathrm{H}), 3.81(\mathrm{~s}, 3 \mathrm{H}), 3.77(\mathrm{~s}, 6 \mathrm{H}), 3.66(\mathrm{~s}, 3 \mathrm{H}), 2.21(\mathrm{~m}, 2 \mathrm{H}), 1.63-1.71(\mathrm{~m}, 4 \mathrm{H}), 1.24(\mathrm{~s} .3 \mathrm{H})$;

${ }^{13} \mathrm{C} \mathrm{NMR}\left(\mathrm{CDCl}_{3}, 100 \mathrm{MHz}\right) \delta 200.0,170.6,159.9,144.8,134.4,105.9,91.5,55.1,52.1,45.6,41.9,34.1$, 25.5, 20.6, 18.1; IR (NaCl plate, $\mathrm{cm}^{-1}$ ): 1740, 1707, 1646, 1606, 1459, 1203, 1152, 1115; X-ray crystallography please see attached cif file.

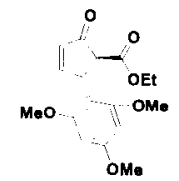

3-Methyl-5-oxo-2-(2,4,6-trimethoxy-phenyl)-cyclopent-3-enecarboxylic acid ethyl ester 14a ${ }^{\mathrm{l}} \mathrm{H}$ NMR $\left(400 \mathrm{MHz}, \mathrm{CDCl}_{3}\right) \delta 6.15(\mathrm{~d}, J=2.1 \mathrm{~Hz}, 1 \mathrm{H}), 6.06(\mathrm{~s}, 1 \mathrm{H}), 5.92(\mathrm{~s}, 1 \mathrm{H}), 4.92(\mathrm{br} . \mathrm{s}, 1 \mathrm{H})$, $4.18(\mathrm{~m}, 2 \mathrm{H}), 3.80(\mathrm{~s}, 6 \mathrm{H}), 3.63(\mathrm{~m}, 4 \mathrm{H}), 1.88(\mathrm{~s}, 3 \mathrm{H}), 1.25(\mathrm{t}, J=7.2 \mathrm{~Hz}, 3 \mathrm{H}) ;{ }^{13} \mathrm{C} \mathrm{NMR}\left(\mathrm{CDCl}_{3}, 100\right.$ MHz) $\delta 202.6,182.4,169.9,160.6,159.6,159.2,127.0,106.4,90.7,90.5,61.2,59.1,56.0,55.3,55.2,43.8$, 17.3, 14.2; IR ( $\mathrm{NaCl}$ plate, $\left.\mathrm{cm}^{-1}\right): 2939,1732,1700,1606,1461,1420,1331,1205,1150,1120,1058,1035$, 815; Elemental analysis calculated for $\mathrm{C}_{18} \mathrm{H}_{22} \mathrm{O}_{6}$ : C $64.66 \% ; \mathrm{H} 6.63 \%$. Found : $\mathrm{C} 64.75 \% ; \mathrm{H} 6.52 \%$.

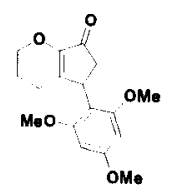




\section{5-(2,4,6-Trimethoxy-phenyl)-3,4,5,6-tetrahydro-2H-cyclopenta[b]pyran-7-one}

16a 'H NMR $\left(400 \mathrm{MHz}, \mathrm{CDCl}_{3}\right) \delta 6.13(\mathrm{~s}, 1 \mathrm{H}), 6.07(\mathrm{~s}, 1 \mathrm{H}), 4.43(\mathrm{~d}, J=5.6 \mathrm{~Hz}, 1 \mathrm{H}), 4.06(\mathrm{~m}, 2 \mathrm{H}), 3.80$ $(\mathrm{s}, 6 \mathrm{H}), 3.63(\mathrm{~s}, 3 \mathrm{H}), 2.66\left(\mathrm{dd}, J_{1}=18.4 \mathrm{~Hz}, J_{2}=6.4 \mathrm{~Hz}, 1 \mathrm{H}\right), 2.47(\mathrm{~d}, J=18.4 \mathrm{~Hz}, 1 \mathrm{H}), 1.98(\mathrm{~m}, 1 \mathrm{H}), 1.90$ $(\mathrm{m}, 1 \mathrm{H}), 1.82-1.90(\mathrm{~m}, 2 \mathrm{H}) ;{ }^{13} \mathrm{C} \mathrm{NMR}\left(\mathrm{CDCl}_{3}, 100 \mathrm{MHz}\right) \delta 200.9,160.0,150.0,148.7,108.0,91.7,91.9$, 66.6, 56.3, 55.2, 40.3, 32.4, 22.0, 21.6; IR ( $\mathrm{NaCl}$ plate, $\left.\mathrm{cm}^{-1}\right): 1705,1649,1604,1461,1224,1202,1151$, 1116; Elemental analysis calculated for $\mathrm{C}_{17} \mathrm{H}_{20} \mathrm{O}_{5}$ : C 67.09\%; $\mathrm{H} 6.62 \%$. Found : $\mathrm{C} 66.97 \%$; $6.80 \%$.

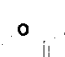

\section{5-Cyclohexyl-3,4,5,6-tetrahydro-2H-cyclopenta[b]pyran-7-one}

16f ${ }^{1} \mathrm{H}$ NMR $\left(400 \mathrm{MHz}, \mathrm{CDCl}_{3}\right) \delta 4.18(\mathrm{~m}, 1 \mathrm{H}), 4.01(\mathrm{~m}, 1 \mathrm{H}), 2.69(\mathrm{~m}, 1 \mathrm{H}), 2.26(\mathrm{~m}, 1 \mathrm{H}), 2.18(\mathrm{~m}, 1 \mathrm{H})$, $2.14(\mathrm{~m}, 1 \mathrm{H}), 1.92(\mathrm{~m}, 2 \mathrm{H}), 1.70(\mathrm{~m}, 6 \mathrm{H}), 1.25-1.32(\mathrm{~m}, 5 \mathrm{H}), 1.09(\mathrm{~m}, 1 \mathrm{H}) ;{ }^{13} \mathrm{C} \mathrm{NMR}\left(\mathrm{CDCl}_{3}, 100 \mathrm{MHz}\right) \delta$ $200.4,151.2,147.2,66.6,42.7,38.6,35.0,31.2,26.4,26.3,26.2,26.1,22.7,21.4$; IR ( NaCl plate, $\left.\mathrm{cm}^{-1}\right)$ : $2922,2851,1708,1644,1117$; Elemental analysis calculated for $\mathrm{C}_{14} \mathrm{H}_{20} \mathrm{O}_{2}: \mathrm{C} 76.33 \% ; \mathrm{H} \mathrm{9.15 \%}$. Found : C $76.06 \%$; $\mathrm{H} 9.03 \%$

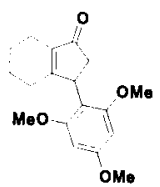

\section{3-(2,4,6-Trimethoxy-phenyl)-2,3,4,5,6,7-hexahydro-inden-1-one}

18a A mixture of stereoisomers was detected by proton NMR, but only the major product could be isolated. The yield range was determined by weighing the mass of the crude product and that of the purified major product. ${ }^{1} \mathrm{H}$ NMR $\left(400 \mathrm{MHz}, \mathrm{CDCl}_{3}\right) \delta 6.70(\mathrm{~m}, 1 \mathrm{H}), 6.16(\mathrm{~s}, 2 \mathrm{H}), 3.80(\mathrm{~s}, 3 \mathrm{H}), 3.78(\mathrm{~s}, 6 \mathrm{H}), 3.45(\mathrm{~m}, 1 \mathrm{H})$, $3.14(\mathrm{~m}, 1 \mathrm{H}), 3.05(\mathrm{~m}, 1 \mathrm{H}), 2.31(\mathrm{~m}, 2 \mathrm{H}), 2.29(\mathrm{~m}, 1 \mathrm{H}), 1.87(\mathrm{~m}, 2 \mathrm{H}), 1.83(\mathrm{~m}, 1 \mathrm{H}), 1.43(\mathrm{~m}, 1 \mathrm{H}) ;{ }^{13} \mathrm{C}$ NMR $\left(\mathrm{CDCl}_{3}, 100 \mathrm{MHz}\right) \delta 207.3,159.6,159.6,142.4,131.6,109.3,100.0,55.6,55.2,41.9,41.6,36.6$, 27.6, 25.5, 21.7; IR ( $\mathrm{NaCl}$ plate, $\left.\mathrm{cm}^{-1}\right): 1710,1645,1221,1193,1152,1115,597$; HRMS calculated for $\mathrm{C}_{18} \mathrm{H}_{22} \mathrm{O}_{4}(\mathrm{M}+\mathrm{H})^{+}$303.1582, Found : 303.1591 .

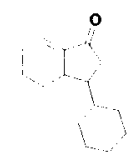

\section{3-Cyclohexyl-2,3,3a,4,5,6-hexahydro-inden-1-one}

$18 f$ A mixture of stereoisomers was detected by proton NMR, but only the major product could be isolated. The yield range was determined by weighing the mass of the crude product and that of the purified major product. ${ }^{1} \mathrm{H}$ NMR $\left(400 \mathrm{MHz}, \mathrm{CDCl}_{3}\right) \delta 6.67(\mathrm{~d}, J=2.8 \mathrm{~Hz}, 1 \mathrm{H}), 2.20-2.35(\mathrm{~m}, 5 \mathrm{H}), 2.04(\mathrm{~m}, 1 \mathrm{H}), 1.77(\mathrm{~m}$, $1 \mathrm{H}), 1.70(\mathrm{~m}, 3 \mathrm{H}), 1.65(\mathrm{~m}, 2 \mathrm{H}), 1.53(\mathrm{~m}, 3 \mathrm{H}), 1.25(\mathrm{~m}, 3 \mathrm{H}), 1.08(\mathrm{~m}, 3 \mathrm{H}) ;{ }^{13} \mathrm{C} \mathrm{NMR}\left(\mathrm{CDCl}_{3}, 100 \mathrm{MHz}\right) \delta$ 205.81, 142.0, 132.0, 47.5, 41.4, 41.0, 40.4, 32.2, 29.4, 28.6, 26.5, 26.4, 26.3, 25.2, 21.7; IR (NaCl plate, $\mathrm{cm}^{-1}$ ): 2922, 2852, 1718, 1651; Elemental analysis calculated for $\mathrm{C}_{15} \mathrm{H}_{22} \mathrm{O}: \mathrm{C} 82.52 \% ; \mathrm{H} 10.16 \%$. Found : C $82.71 \%$; $9.91 \%$ 


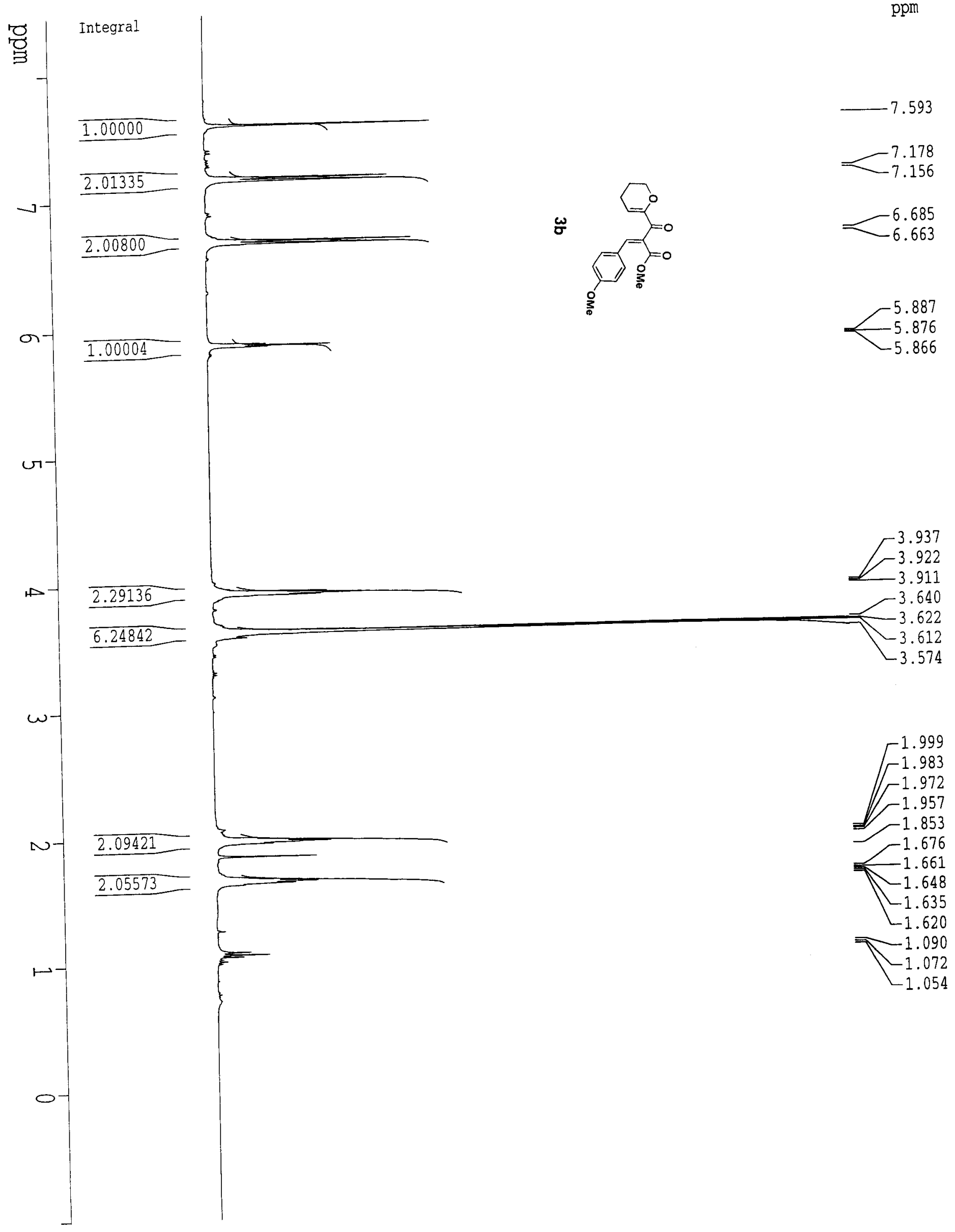




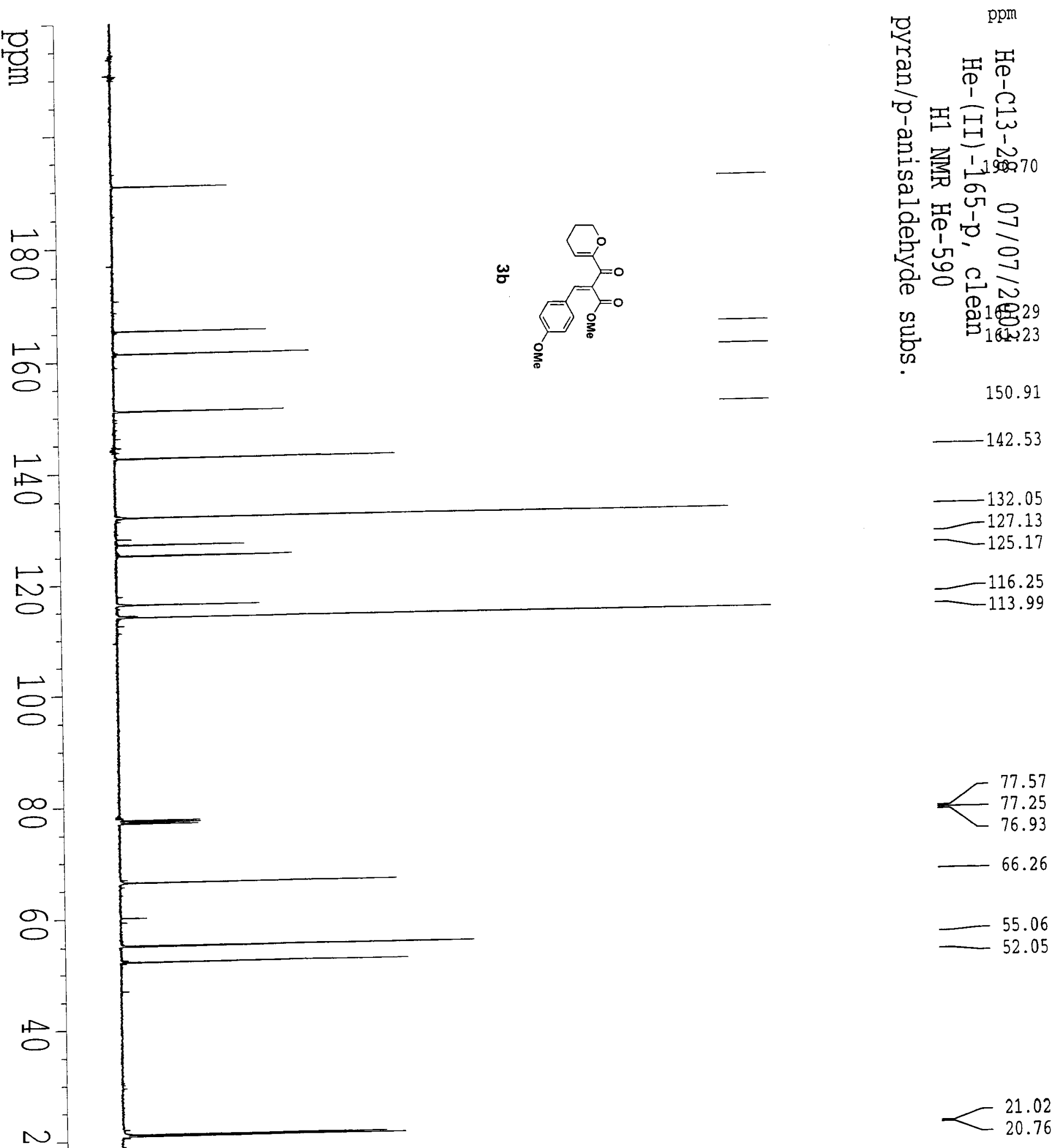




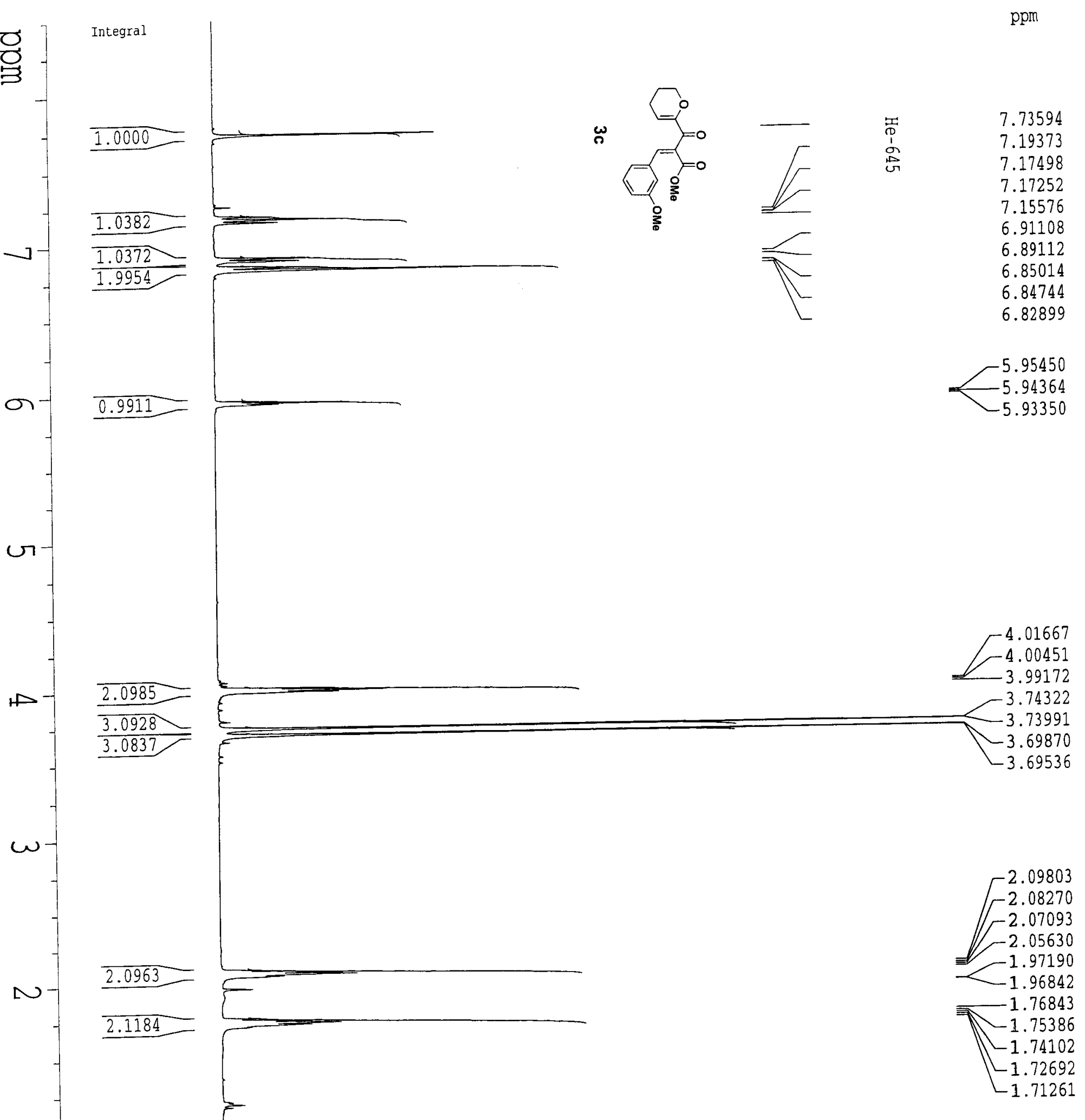



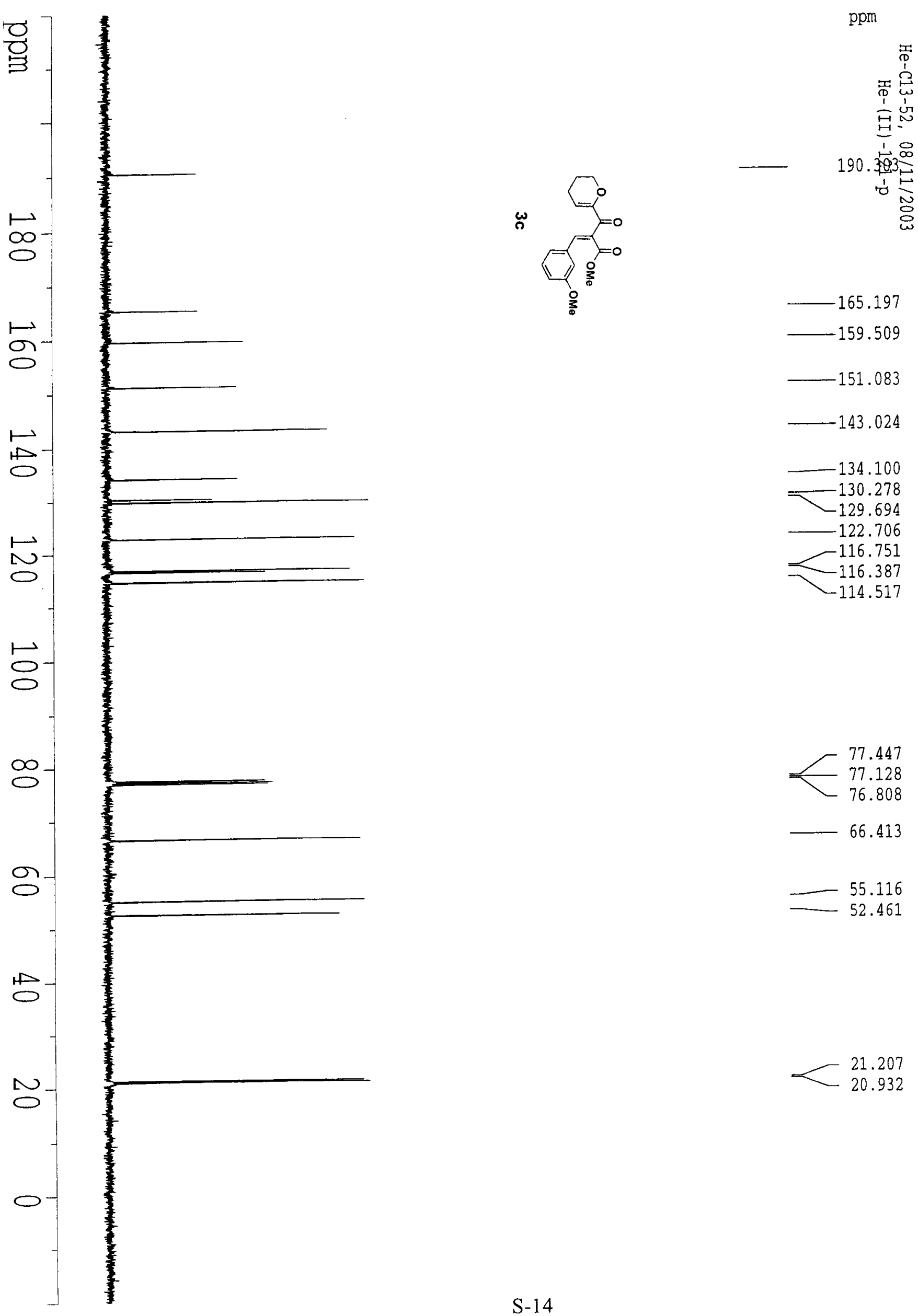

21.207
$\times \quad 20.932$ 


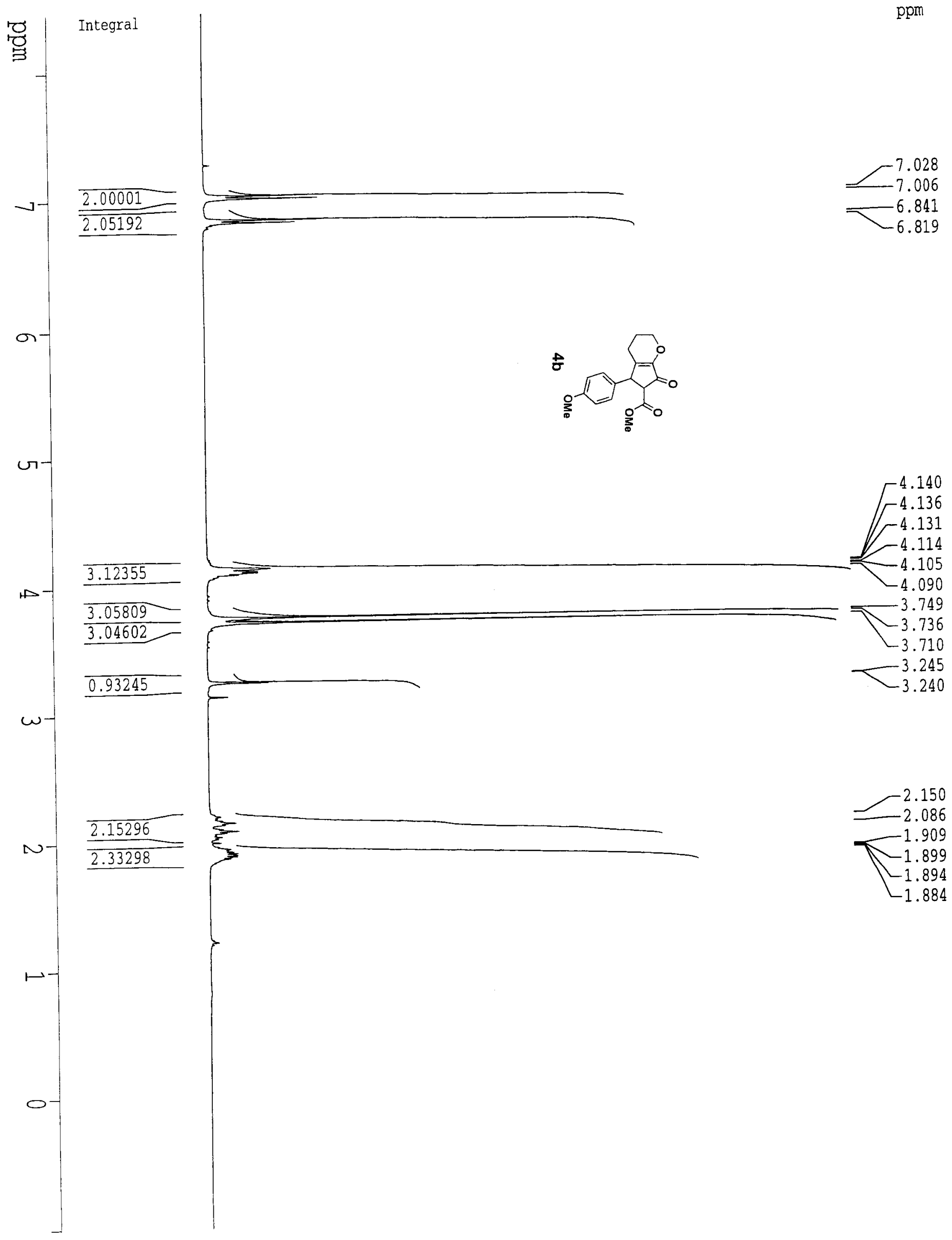




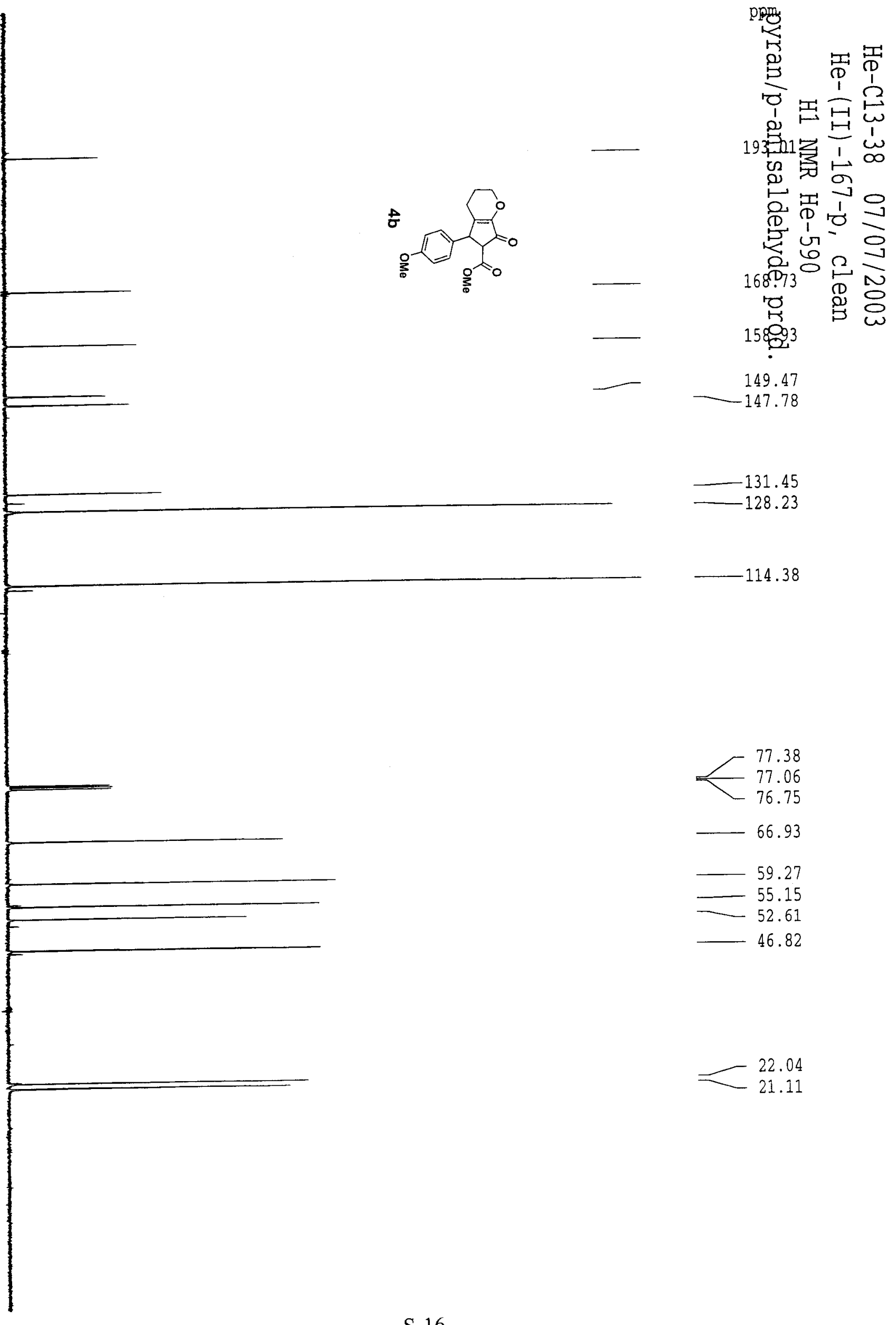




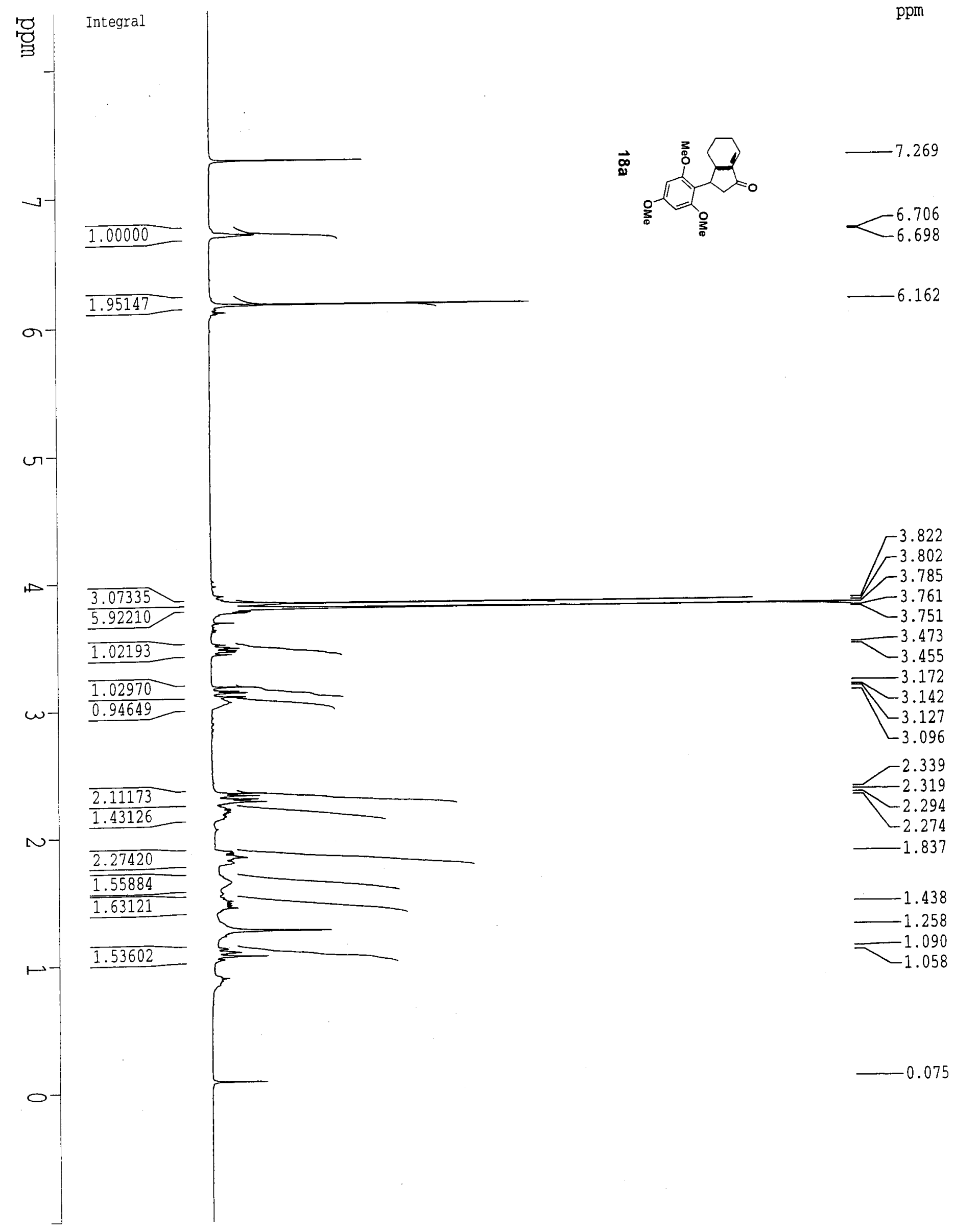


